\title{
Liter per Minute per Microgram per Kilogram
}

National Cancer Institute

\section{Source}

National Cancer Institute. Liter per Minute per Microgram per Kilogram. NCI Thesaurus.

Code C120757.

A unit of concentration equal to liter per minute divided by microgram per kilogram. 